\title{
A Case of Acute Vestibular Neuritis with Periodic Alternating Nystagmus
}

\author{
Se Won Jeong ${ }^{1}$, Jeon Mi Lee ${ }^{2}$, Sung Huhn Kim², and Jung Hyun Chang ${ }^{1}$ \\ ${ }^{1}$ Department of Otorhinolaryngology-Head and Neck Surgery, National Health Insurance Service Ilsan Hospital, Goyang; and \\ ${ }^{2}$ Department of Otorhinolaryngology, Yonsei University College of Medicine, Seoul, Korea
}

\section{주기성 교대 안진을 동반한 전정 신경염 1예}

정세원 ${ }^{1} \cdot$ 이전미 ${ }^{2} \cdot$ 김성헌 ${ }^{2} \cdot$ 장정현 ${ }^{1}$

국민건강보험 일산병원 이비인후과, ${ }^{1}$ 연세대학교 의과대학 이비인후과학교실 ${ }^{2}$

\author{
Received August 13, 2016 \\ Revised October 13, 2016 \\ Accepted November 3, 2016 \\ Address for correspondence \\ Jung Hyun Chang, MD, PhD \\ Department of Otorhinolaryngology- \\ Head and Neck Surgery, \\ National Health Insurance \\ Service Ilsan Hospital, \\ 100 Ilsan-ro, Ilsandong-gu, \\ Goyang 10444, Korea \\ Tel $+82-31-900-0972$ \\ Fax $+82-31-900-0972$ \\ E-mail manbang5@naver.com
}

Detection of nystagmus is an important diagnostic clue in patients with acute vertigo. Patients with peripheral disorders exhibit nystagmus with a constant direction whereas those with central disorders exhibit nystagmus with changes in direction with or without gaze fixation. Periodic alternating nystagmus (PAN) is a horizontal or horizontal-rotary jerk-type nystagmus that reverses its direction with time. PAN is typically observed in patients with central disorders, such as cerebellar or pontomedullary lesions, but it is also observed in patients with peripheral disorders, albeit rarely. Here we report a rare case of a 58-year-old patient with vertigo with PAN, which was initially suspected as a central disorder, but eventually diagnosed as a peripheral vestibular disorder. We investigated the characteristics and mechanisms of peripheral PAN in this case. The absence of central disorder symptoms, visual suppression of PAN, normal oculomotor findings, and transient persistence are important diagnostic clues for differentiating peripheral from central PAN. Korean J Otorhinolaryngol-Head Neck Surg 2018;61(3):151-5

Key Words Periodic alternating nystagmus - Vestibular neuritis · Vestibule of the ear.

\section{Introduction}

Periodic alternating nystagmus (PAN) is a persistent horizontal or horizontal-rotary jerk-type nystagmus that reverses its direction in intervals of $1-6$ minutes. It is known to have resting periods of 4-20 seconds between switches, ${ }^{1)}$ although the periodicity and quiescence are not regular in all cases. This condition is typically observed either in patients with a complaint of acute vertigo in central nervous system disorders, especially those of the pontomedullary area, or in patients with the congenital form of PAN. ${ }^{2,3)}$ However, very rarely, peripheral vestibular disorders such as labyrinthitis or Meniere's

This is an Open Access article distributed under the terms of the Creative Commons Attribution Non-Commercial License (http://creativecommons.org/licenses/by-nc/4.0) which permits unrestricted non-commercial use, distribution, and reproduction in any medium, provided the original work is properly cited. disease presenting PAN have been also reported. ${ }^{4)}$

Here we report an uncommon case of a patient with acute vertigo presenting with PAN, which was eventually diagnosed as a peripheral vestibular disorder-vestibular neuritis-without other central disorders. We investigated the characteristics and mechanisms of peripheral PAN with a review of literature.

\section{Case}

A 58-year-old woman visited our clinic with aggravated vertigo with an onset duration of 2 months. A review of her medical history revealed mastoidectomy because of chronic otitis media in the right ear 30 years ago and no other medical conditions. The patient suffered a spinning type of vertigo lasting 2-3 minutes, with no other otological symptoms such 
as hearing loss or fullness of the ear. Vertigo was relieved with rest, but it soon aggravated whenever the patient changed position. The ear drum was normal, with no signs of infection. Spontaneous or gaze-evoked nystagmus was not detected, but when the head rolling test was administered, geotropic nystagmus stronger in the right-sided position was observed. The patient was tentatively diagnosed with benign paroxysmal positional vertigo of right-sided lateral semicircular canalolithiasis, which was treated with the Barbecue maneuver. However, when the symptoms did not improve significantly, the patient was hospitalized for conservative treatment. After 2 days of hospitalization, the pattern of nystagmus was observed to have changed. The patient exhibited spontaneous nystagmus beating to one side, followed by a change in direction to the opposite side, with a quiescence period of 4-6 seconds between reversals (Fig. 1). Each unidirectional cycle lasted 15-20 seconds, and the mean maximal slow phase velocity was $4-12 \%$ second. This periodicity was repeated, which suggested that either direction of nystagmus could be started first. The pattern of the nystagmus did not change during the Dix-Hallpike or head rolling tests, indicating its independence from change in position.

Neurological examination, including cerebellar function test and brain magnetic resonance imaging, was performed to evaluate the possibility of central origin of nystagmus; however, the findings revealed no definite abnormalities. Evaluation for tumor biomarkers or neuromyelitis optica antibodies to rule out paraneoplastic syndrome, revealed negative findings. Vestibular function tests were then performed. Smooth pursuit and saccadic eye movement exam revealed normal vestibule-ocular reflex, with minor variations due to spontaneous nystagmus. Bithermal caloric test was performed to in-
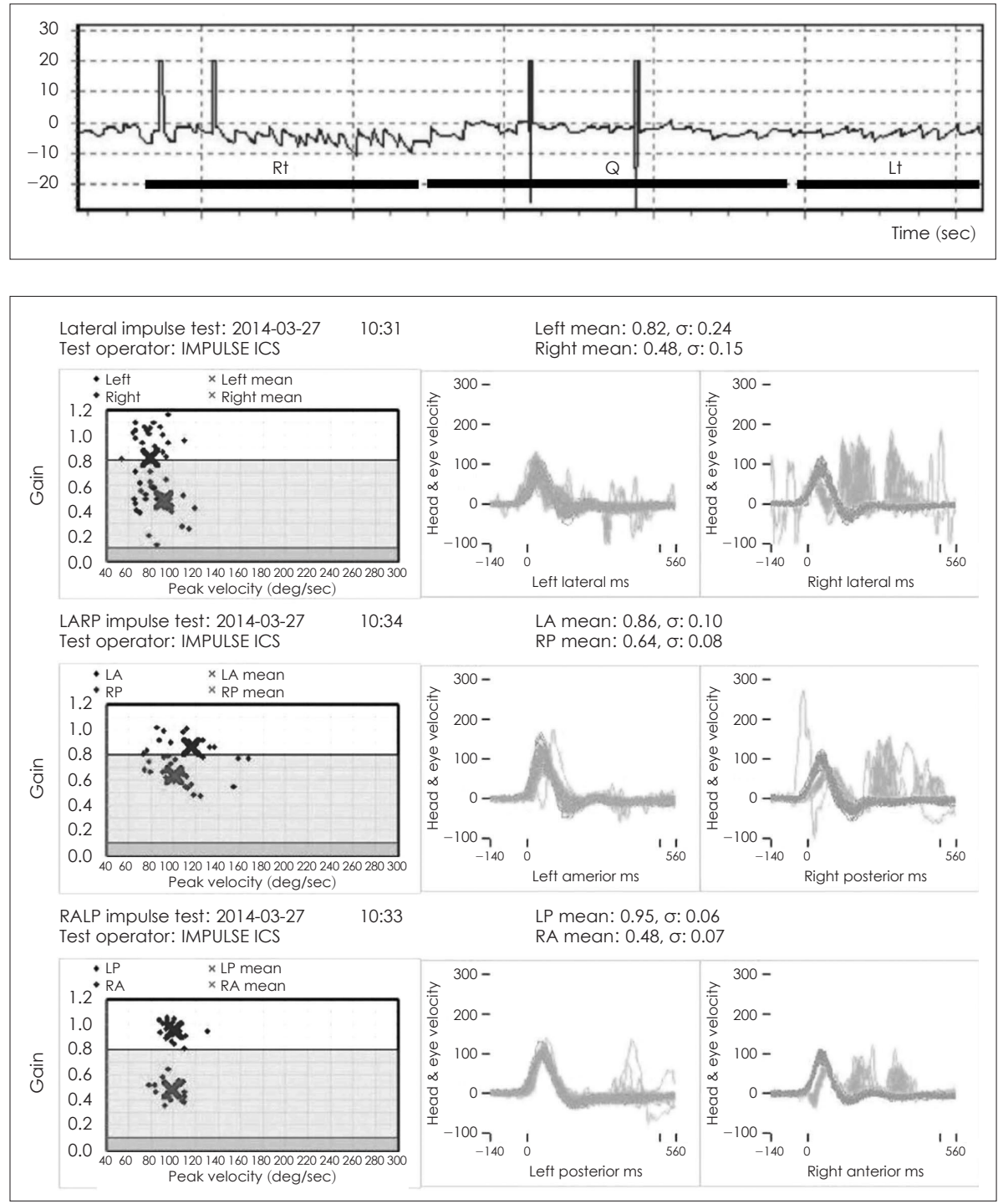

Fig. 1. A 30-second trace of spontaneous nystagmus in the dark. Right horizontal beating nystagmus (Rt) transformed to left horizontal beating nystagmus (Lt) after 12 seconds of quiescence ( $Q$ ). The trace represents horizontal eye movement (degree).

Fig. 2. Video head impulse test was performed during the hospital stay. Right sided canals showed decreased gain and following catch-up saccade during the test, suggesting peripheral vestibulopathy in right side. LARP: left anterior right posterior, LA: left anterior, RP: right posterior, RALP: right anterior left posterior. 
vestigate the canal function. It was impossible to calculate the canal paresis and the directional paresis value because direction-changing nystagmus remained still during the test. However, nystagmus was well suppressed during the caloric test with visual fixation. Video head impulse test was performed to evaluate the function of each canal. Right sided canals showed decreased gain with following catch-up saccades, suggesting peripheral vestibulopathy (Fig. 2). Cervical vestibular evoked myogenic potential test revealed no response in the right ear and a threshold of $85 \mathrm{~dB}$ in the left ear. Upon posturography, the patient constantly fell in conditions 4, 5, and 6 (Fig. 3A), and decreased vestibular function in sensory analysis (Fig. 3B). Consequently, the patient was diagnosed with acute vestibular neuritis affecting the right side, rather than a central disorder. The patient continued to receive conservative care with vestibular rehabilitation therapy.

After 6 days from its first observation, PAN had resolved completely, while spontaneous nystagmus beating to left side, which was constant while changing position, persisted. Head thrust test revealed corrective saccade movement in the right thrust position, which supported our diagnosis of vestibular neuritis. After 2 weeks of vestibular rehabilitation therapy, the patient exhibited improvement in her symptoms and has not complained of relapse in follow-up evaluations till date. Follow-up serial posturographic findings revealed improvement as well (Fig. 3).

\section{Discussion}

PAN is differentiated as either an acquired condition or a type of congenital nystagmus. Congenital PAN often presents during the first 6 months after birth and has been reported to be associated with sensory defects such as albinism. ${ }^{5}$ Acquired PAN, although less common, is equally important to identify because of its association with central nervous system disorders involving the pontomedullary area, caudal brainstem, or vestibulocerebellum. ${ }^{1)}$ Anticonvulsant medications, hepatic encephalopathy, and trauma and the consequent vision loss are also known to cause PAN. ${ }^{5}$ )

However, PAN has also been observed in patients with pe-

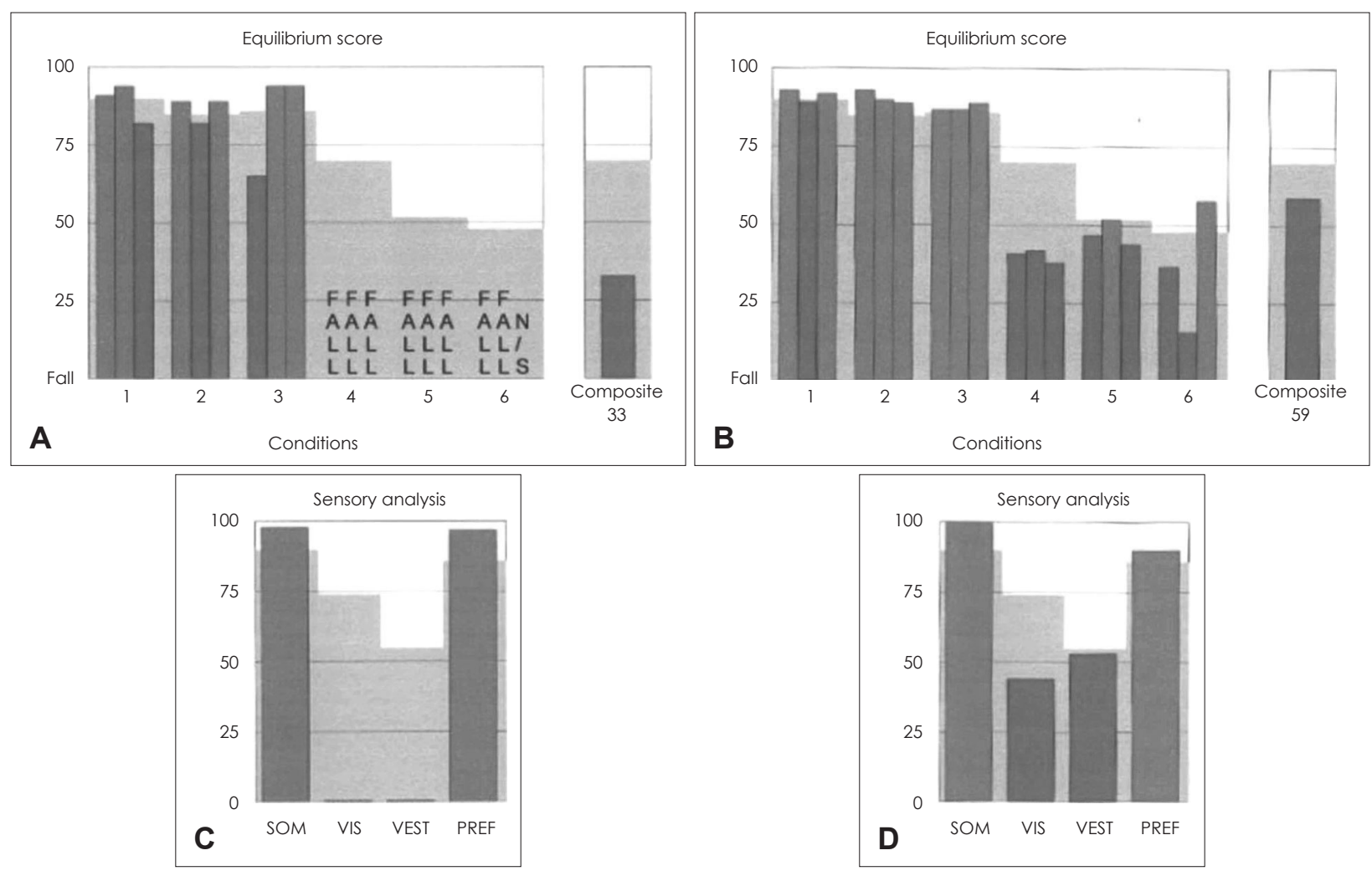

Fig. 3. Serial dynamic posturographic findings of the patient. At initial posturography, the patient constantly fell in conditions 4,5 , and 6 and exhibited a composite score of $33(\mathrm{~A})$. Posturographic findings at 2 months after vestibular rehabilitation (B). Although the patient still exhibited instability in conditions 4,5 , and 6 , the composite score (59) was much improved compared to that at initial posturography. Sensory analysis information from initial posturography. Vestibular and vision dysfunction was noted (C). After 2 months with vestibular rehabilitation, vestibular and vision function showed improvement (D). SOM: somatosensory, VIS: vision, VEST: vestibular, PREF: preference. 
ripheral vestibular disorders, albeit rarely. Kim, et al. ${ }^{2)}$ reported 10 patients with peripheral vestibular disorder presenting PAN and defined the distinguishing characteristics of peripheral and central PAN. Central PAN can be identified by the presence of symptoms of central disorders, such as ataxia, motor weakness, dysarthria, lethargy, or abnormal cerebellar function findings. ${ }^{6)}$ In addition, central PAN persists for over several months or is permanent, while peripheral PAN is thought to be a temporary condition. All cases of peripheral PAN reported till date, including the present case, progressed to unidirectional spontaneous nystagmus within 48 hours. ${ }^{2)}$ Visual suppression of nystagmus and normal oculomotor findings are other clinical features of peripheral PAN. ${ }^{2)}$ In contrast, PAN associated with central disorders are generally not influenced by visual fixation. ${ }^{5)}$

Similar to the clinical features, the pathomechanisms underlying central and peripheral PAN are thought be distinct. The mechanisms of central PAN are still unclear, but the findings of a previous animal study suggest that it could be associated with inhibitory inputs to velocity storage mechanisms. ${ }^{7)}$ A previous study reported that impairment of the cerebellar nodulus and uvula resulted in the removal of inhibition of the velocity storage mechanism, thus prolonging the duration of rotationally-induced nystagmus. ${ }^{5)}$ Additionally, damage to the cerebellar flocculus and para flocculus prevents visual fixation, which eliminates the activity of the velocitystorage mechanism. ${ }^{5}$ These findings explain not only the suppression of nystagmus with visual fixation in peripheral PAN, but also the reason for central PAN being observed in the dark. On the other hand, the mechanisms resulting in peripheral PAN are thought to occur during the transition period when irritative nystagmus changes to paralytic nystagmus. ${ }^{2)}$ In the irritative phase, unilateral vestibular nuclei are stimulated, and nystagmus is observed in the direction of the stimulated side. Then, as input to the vestibular nuclei decreases, the direction of nystagmus changes to the contralateral side, with paralysis of the peripheral vestibular system. However, Cunha Ribeiro and Pérez-Fernandes ${ }^{8}$ reported a case of PAN due to impairment of central visual stabilization mechanisms and deficient visual input and fixation caused by ophthalmological antecedent, which suggested other possible causes of PAN, apart from exact peripheral or central reasons. ${ }^{8)}$

Although our patient in the present case was consequently diagnosed with peripheral vestibular disorder, PAN is commonly observed as a significant manifestation of central lesions and certainly warrants initial tentative diagnosis of central lesions. According to previous reports, including a recent systemic study of central paroxysmal positional nystagmus, acquired PAN can occur in patients with cerebellar diseases, such as degeneration, mass lesions, Arnold-Chiari malformation, Creutzfeldt-Jakob disease, multiple sclerosis, and ataxia telangiectasia, and brain stem infarctions." ${ }^{9}$ Specifically, most patients with central paroxysmal positional nystagmus were reported to exhibit involvement of the cerebellar nodulus or uvula. Possible diagnosis of paraneoplastic syndrome has to be considered in patients with PAN, particularly in cases of nystagmus with a vertical component. ${ }^{10,11)}$ In some patients, paraneoplastic syndromes have been reported to present PAN, thought to be related to the dysfunction of Purkinje cells in paraneoplastic flocculus and paraflocculus. ${ }^{10)}$ Given the high incidence of PAN as a central lesion, we initially performed neurological examination, including cerebellar function test and brain magnetic resonance imaging, as well as additional laboratory evaluations for paraneoplastic syndrome in order to exclude the possibility of a central lesion. $^{12)}$

After differential diagnosis, if the patient is diagnosed with a peripheral vestibular disorder, conservative care with vestibular rehabilitation therapy could be the appropriate treatment. Upon treatment, our patient exhibited improvement in symptoms and improved serial posturographic findings at followup. Both congenital and acquired PAN can often be resolved by treatment with baclofen. Because of its inhibitory action on vestibular rotational responses, baclofen, a gamma-aminobutyric acid-nergic drug, effectively resolves acquired PAN. However, the cause of PAN rather than its treatment seems clinically more important."

In conclusion, PAN is a rare physical finding, difficult to detect because of its transitory nature. It could be easily overlooked in cases where nystagmus is not observed for a sufficient period. Although PAN is generally known to be a significant manifestation of central lesions, ophthalmologists need to be aware that peripheral vestibular disorders could also present atypical physical findings such as PAN. Suppression of nystagmus with visual fixation and temporary persistence of PAN without other central neurologic signs can be important diagnostic signs for differentiating between peripheral and central PAN. The findings of the present case and literature review suggest the possibility of peripheral vestibular disorders in patients with vertigo presenting PAN. 


\section{REFERENCES}

1) Davis DG, Smith JL. Periodic alternating nystagmus. A report of eight cases. Am J Ophthalmol 1971;72(4):757-62.

2) Kim SH, Chung WK, Kim BG, Hwang CS, Kim MJ, Lee WS. Periodic alternating nystagmus of peripheral vestibular origin. Laryngoscope 2014;124(4):980-3.

3) Norré ME, Puls T. Nystagmus alternans. Acta Otorhinolaryngol Belg 1981;35(2):198-206.

4) Murofushi T, Chihara Y, Ushio M, Iwasaki S. Periodic alternating nystagmus in Meniere's disease: the peripheral type? Acta Otolaryngol 2008;128(7):824-7.

5) Shallo-Hoffmann J, Riordan-Eva P. Recognizing periodic alternating nystagmus. Strabismus 2001;9(4):203-15.

6) Kim MB, Ban JH. Acute central vertigo. Korean J OtorhinolaryngolHead Neck Surg 2014;57(8):504-10.

7) Waespe W, Cohen B, Raphan T. Dynamic modification of the vestibuloocular reflex by the nodulus and uvula. Science 1985;228(4696):
199-202.

8) Cunha Ribeiro D, Pérez-Fernandes N. Periodic alternating nystagmus. A case report. Acta Otorrinolaringol Esp 2016;67(1):e1-3.

9) Choi JY, Kim JH, Kim HJ, Glasauer S, Kim JS. Central paroxysmal positional nystagmus: characteristics and possible mechanisms. Neurology 2015;84(22):2238-46.

10) Choi SY, Park SH, Kim HJ, Kim JS. Paraneoplastic downbeat nystagmus associated with cerebellar hypermetabolism especially in the nodulus. J Neurol Sci 2014;343(1-2):187-91.

11) Eggers SD, Pittock SJ, Shepard NT, Habermann TM, Neff BA, Klebig RR. Positional periodic alternating vertical nystagmus with PCA-Tr antibodies in Hodgkin lymphoma. Neurology 2012;78(22): 1800-2.

12) Lee HJ, Bae SH, Lee JM, Kim SH. A case of central origin vertigo in breast cancer with brain metastasis. Korean J Otorhinolaryngol-Head Neck Surg 2013;56(9):584-7. 